\title{
A Glycine-Rich Protein Encoded by Sulfur-Deficiency Induced Gene is Involved in the Regulation of Callose Level and Root Elongation
}

\author{
Anna Znój, Katarzyna Zientara-Rytter, Pawel Sęktas, Grzegorz Moniuszko, \\ Agnieszka Sirko and Anna Wawrzyńska ${ }^{1}$
}

\begin{abstract}
Glycine-rich proteins (GRPs) with characteristic repetitive glycine stretches are ubiquitous in organisms of all Kingdoms and have distinct functions. It is believed that Gly-rich domains serve mainly for interactions with other proteins. Previously, we identified the tobacco UP30 gene as strongly upregulated by sulfur deficiency. It encodes a protein highly similar to cdiGRP involved in tobacco defense response by elevating cell wall callose deposits thus blocking systemic movement of viruses. The closest Arabidopsis thaliana homologue of UP30 is GRP3 (At2g05520). Here we report that GRP-3 is induced in Arabidopsis seedlings in both sulfur and nitrogen deficiency conditions. The transgenic Arabidopsis plants either overexpressing or with silenced GRP-3 expression tend to have longer roots especially in the conditions of sulfur deficiency. The effect could be reduced by the addition of auxin to the media. Moreover, we observed the increased callose deposition in both Arabidopsis lines suggesting its negative effects on shoot-to-root movement of auxins in nutrient deficient conditions.
\end{abstract}

Glycine-rich proteins (GRPs) are characterized by the existence of several domains with little sequence conservation but highly enriched in glycine. Although the first genes encoding GRPs have been isolated from plants, proteins with characteristic repetitive glycine stretches exist in diverse organisms from cyanobacterias to animals (reviewed in Sachetto-Martins et al. 2000). The first reports described plant GRPs as cell wall associated proteins (Showalter, 1993), however since then many other GRPs with different domain organizations, sub-cellular localizations as well as tissue specificity of expression were demonstrated. This clearly indicated that these proteins must be implicated in several independent physiological processes, though the specific functions of several so far characterized GRPs remain speculative. The diversity of GRPs led even to the concept that they should rather not be considered as a family but as a wide group of proteins sharing a common motif (Sachetto-Martins et al. 2000). The presence of Gly-rich highly flexible domains may act as a molecular glue in the protein-protein interactions. It is therefore quite probable that GRPs are components of different

A. Znój • K. Zientara-Rytter • P. Sęktas • G. Moniuszko • A. Sirko $(\bowtie) \cdot A$. Wawrzyńska

Institute of Biochemistry and Biophysics, Polish Academy of Sciences

Pawińskiego 5A St, 02-106 Warsaw, Poland

e-mail: asirko@ibb.waw.pl 
multimolecular complexes, including homo- or hetero-dimerization between family members (Sachetto-Martins et al. 2000).

The UP30 gene encoding protein belonging to GRP family has been identified as one of the strongly upregulated genes in the leaves of tobacco plants transferred for two days to the sulfur deficient medium (Wawrzynska et al. 2005). UP30 is a small protein containing Gly-rich, Tyr-rich and Pro-rich domains and a characteristic Cys-rich motif [C-X3-CC-X6-C-X2-CC-] (Lewandowska et al. 2005). Subsequent experiments indicated that the protein fusion of UP30 and YFP is located in the cell wall (Fig.1A). The yeast two hybrid system allowed us to exclude the UP30-UP30 homodimerization (Fig. 1B); the interactions with other members of the GRP family were not checked.

UP30 belongs to Class II from the eight classes that the plant GRPs has been divided into based on the domain composition (Mangeon et al. 2010). Class II members have (beside optional signal peptide at the N-terminus) the Gly-rich and Cys-rich domains. Previously identified tobacco protein from the same class (cdiGRP) was proposed to be involved in plant defense. The gene encoding this protein is induced by cadmium and the elevated level of cdiGRP has been suggested to block the systemic spread of the turning vein-clearing tobamovirus (Ueki and Citovsky 2002) due to increasing the callose deposition in the plant cell wall (Ueki and Citovsky 2005). Interestingly, UP30 shares $81 \%$ amino acid sequence identity with cdiGRP allowing us to assume both proteins are functionally similar. The closest Arabidopsis thaliana homologue of UP30 is GRP-3. Both proteins have very similar domains/regions organization despite relatively low sequence conservation (only $29 \%$; Fig. 2).

The GRP-3 (At2g05520) gene was previously shown to be expressed in stems and leaves and only poorly in the roots, immature seed pods and flowers (de Oliveira et al. 1990). Such pattern of expression resembles the expression of UP30; the UP30 mRNA was detected mostly in the leaves (Lewandowska et al. 2005). Likewise, the localization of GRP-3 in the apoplast (Gramegna et al. 2016) was an additional suggestion for the functional homology between UP30 and GRP-3. The GRP-3 protein interacts through its Cys-rich C-terminal motif with the cell wall receptor protein kinase WAK1. The binding of GRP-3 to WAK1 is critical for the structural integrity of the multimeric complex representing the activated signalosome of pathogen response (Park et al. 2001). On the other hand, GRP-3 acts as negative regulator of both elicitor-activated signaling cascade and response to wounding. It was proposed that GRP-3 may function in restoring the plant immune system to the baseline at the appropriate time after the pathogen attack (Gramegna et al. 2016).

To assay the function of GRP-3 during sulfur deficiency we have constructed the transgenic Arabidopsis plants constitutively overexpressing GRP-3 (OX9 lines) and plants with silenced $G R P-3$ due to constitutive expression in the antisense orientation (KD10 lines). The level of GRP-3 mRNA in the transgenic plants was confirmed with semiquantitative RT-PCR. Additionally, the expression level was monitored in plants grown in S- or N-deficient conditions. The level of GRP-3 transcript was induced in Sand $\mathrm{N}$ - conditions in the wild-type seedlings, it was high in the OX9 line and low (or undetected) in KD10 line (Fig. 3).

Interestingly, monitoring the growth of the seedlings in sulfur- deficient (S-), and sufficient (ctrl) media indicated that both overexpressors as well as knock-downs of 
GRP-3 have longer roots that the wild-type, especially in the conditions of sulfur deficiency (Fig. 4).

Tobacco cdiGRP represents one of the factors that regulates callose accumulation in the plant vasculature (Ueki and Citovsky 2005). Callose is a 1,3- $\beta$-d-glucan, synthesized by callose synthase and degraded by $1,3-\beta$-d-glucanase, which has been shown to accumulate around the neck region of the plasmodesmata. It is supposed to regulate the traffic through these channels and cell-to-cell communication. Therefore, in the next step we verified the level of callose in the GRP-3 transformants. Surprisingly, higher level of callose was observed in the tissue of both OX9 and KD10 plants as compared to the wild type (Fig. 5A). It was especially significant for the stem and root vascular tissue, especially at the plasmodesmata region (Fig. 5B).

The plasmodesmata is a specific channel structure in plants that spans the cell wall connecting neighboring cells to allow cytoplasmic exchange and communication. The plasmodesmata contribution to auxins movement, their role as potential sites of receptor signaling and the impact of callose deposition in plasmodesmata on auxins function has been recently discovered (Jackson 2015). Auxins reduce the growth of roots by two types of mechanisms, either by reducing the extend of root growth (IAA, NAA) or the cell production rate (2,4-D) (Rahman et al. 2007). Therefore, we assumed that the OX9 and KD10 transformants have longer roots than the wild type in nutrient deficient conditions because the shoot-to-root movement of auxins in such plants is negatively affected due to extensive callose deposition in their plasmodesmata (Fig. 5). It has been previously shown that Arabidopsis plants grown in S- conditions have lower level of auxins, as indicated by the activity of the auxin response marker DR5::GUS in the roots (Dan et al. 2007). To check this we decided to compare the effect of NAA on the root elongation of the seedlings grown in S-conditions (Fig. 6). In this experiment the OX9 lines were used as plants overexpressing GRP-3 and three T-DNA insertion SALK lines (SALK_084781, SALK_084877 and SALK_012941C) were used as KO grp-3 mutants. These lines had no detectible transcript corresponding to GRP-3 (not shown). The results shown in Fig. 6 indicated that addition of NAA to the S-deficient media negatively affected root length in all lines; however, the difference in root lengths observed between the wild-type seedlings and the OX9 transformants and grp3 insertional mutants was strongly reduced in the presence of auxin.

In conclusion, we have demonstrated that the GRP-3 protein encoded by the sulfur deficiency induced gene modulates the root length of the Arabidopsis seedlings in nutrient deficient conditions. The longer roots were observed in both, seedlings with increased level of GRP-3 as well as with the reduced or abolished expression of GRP-3. An unbalanced level of GRP-3 results in increased callose deposition in plasmodesmata. Our findings are supported by the reports from other laboratories, where on one hand, the elevated expression of cdiGRP in tobacco resulted in callose accumulation (Ueki and Citovsky 2002, 2005), while on the other hand, the Arabidopsis grp-3 mutants were reported to have higher level of callose than the wild-type in response to different elicitors (Gramegna et al. 2016). Results of the experiments presented in this work prompt us to hypothesize that GRP-3 is involved in the regulation of root growth in response to nutrient status of the plants through influencing auxin movement due to affecting callose deposits in plasmodesmata. 
Acknowledgments We thank to Dimitris Bouranis, Agricultural University of Athens, Greece for the protocol of callose staining. This work was supported by the grant from the Polish Ministry of Science and Higher Education (W16/7.PR/2011).

\section{References}

Dan H, Yang G, Zheng ZL (2007) A negative regulatory role for auxin in sulphate deficiency response in Arabidopsis thaliana. Plant Mol Biol 63:221-235

De Oliveira DE, Seurinck J, Inze D, Van Montagu M, Botterman J (1990) Differential expression of five Arabidopsis genes encoding glycine-rich proteins. Plant Cell 2:427-436

Gramegna G, Modesti V, Savatin DV, Sicilia F, Cervone F, De Lorenzo G (2016) GRP-3 and KAPP encoding interactors of WAK1 negatively affect defense responses induced by oligogalacturonides and local response to wounding. J Exp Bot 67:1715-1729

Jackson D (2015). Plasmodesmata spread their influence. F1000Prime Rep 7:25

Lewandowska M, Wawrzynska A, Kaminska J, Liszewska F, Sirko A (2005) Identification of novel proteins of Nicotiana tabacum regulated by short term sulfur starvation. In: Saito K, De Kok LJ, Stulen I, Hawkesford MJ, Schnug E, Sirso A, Rennenberg H (eds), Sulfur Transport and Assimilation in Plants in the Postgenomic Era, pp 153-156, Backhuys Publishers, Leiden

Mangeon A, Junqueira RM, Sachetto-Martins G (2010) Functional diversity of the plant glycinerich proteins superfamily. Plant Signal Behav 5:99-104.

Park AR, Cho SK, Yun UJ, Jin MY, Lee SH, Sachetto-Martins G, Park OK (2001) Interaction of the Arabidopsis receptor protein kinase Wak1 with a glycine-rich protein AtGRP-3. J Biol Chem 276:26688-26693

Rahman A, Bannigan A, Sulaman W, Pechter P, Blancaflor EB, Baskin TI (2007) Auxin actin and growth of the Arabidopsis thaliana primary root. Plant J 50:514-528

Sachetto-Martins G, Franco LO, de Oliveira DE (2000) Plant glycine-rich proteins: a family or just proteins with a common motif? Biochim Biophys Acta 1492:1-14

Showalter AM (1993) Structure and function of plant cell wall proteins. Plant Cell 5:9-23

Ueki S, Citovsky V (2002). The systemic movement of a tobamovirus is inhibited by a cadmiumion-induced glycine-rich protein. Nat Cell Biol 4:478-486

Ueki S, Citovsky V (2005). Identification of an interactor of cadmium ion-induced glycine-rich protein involved in regulation of callose levels in plant vasculature. Proc Natl Acad Sci U S A 102:12089-12094

Wawrzynska A, Lewandowska M, Hawkesford MJ, Sirko A (2005) Using a suppression subtractive library-based approach to identify tobacco genes regulated in response to shortterm sulphur deficit. J Exp Bot 56:1575-1590 


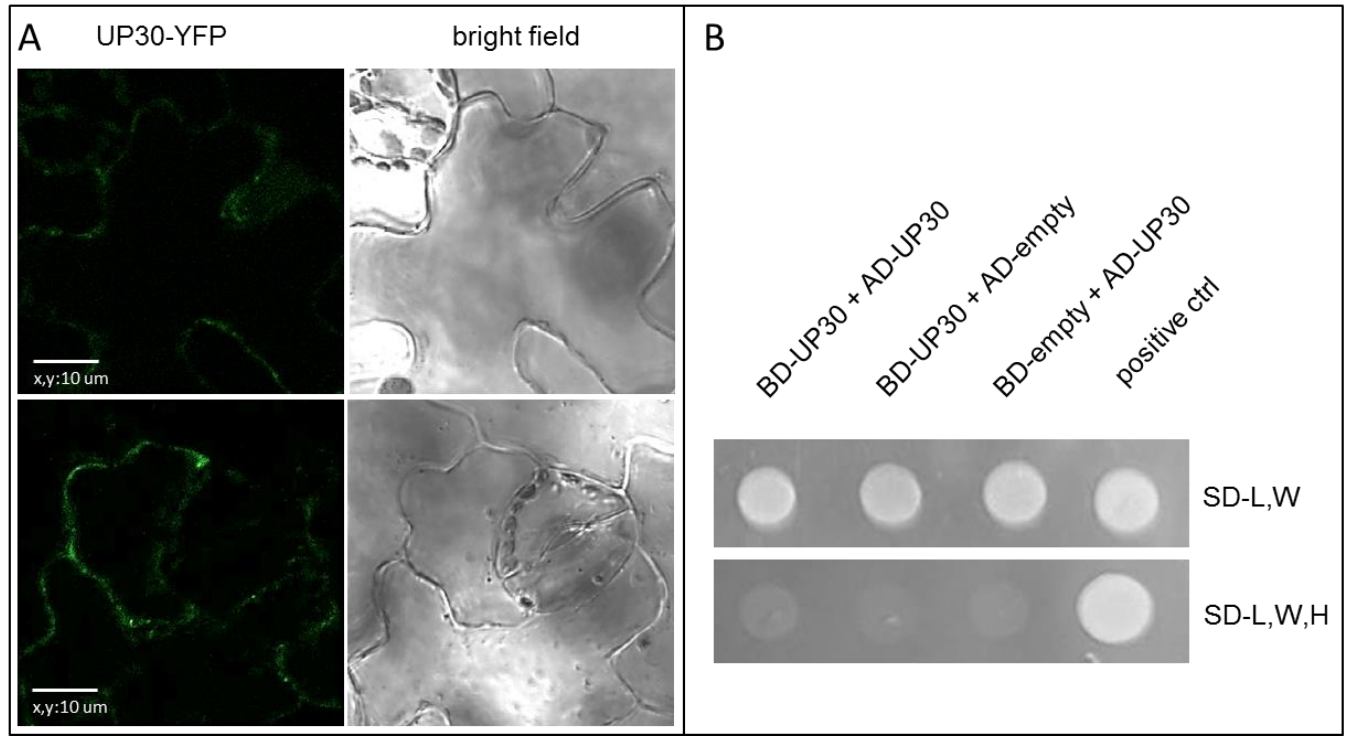

Fig. 1 UP30 protein localize in the cell wall of leaf epidermal cells when transiently expressed in $N$. benthamiana (A) and does not form dimers in the yeast two hybrid test (B).

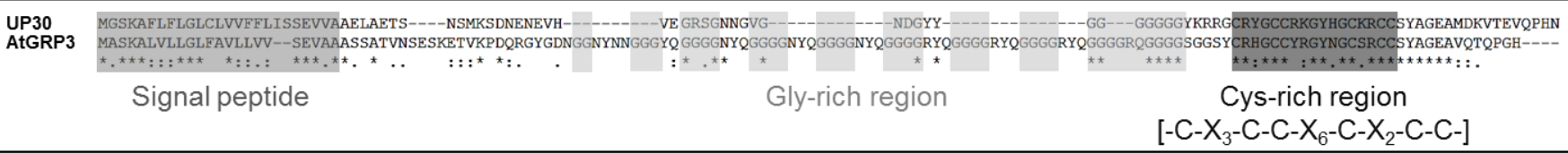

Fig. 2 Amino-acid alignment of tobacco UP30 and GRP-3. Characteristic domains are marked 


\begin{tabular}{|lllllllllll|}
\hline & \multicolumn{3}{c}{ ctrl } & \multicolumn{9}{c}{ S- } & & \multicolumn{3}{c|}{ N- } \\
GRP3 & OX9 & KD10 & wt & OX9 & KD10 & wt & OX9 & KD10 & wt \\
TUA3 & & & & & & & & & \\
\hline
\end{tabular}

Fig. 3 The level of GRP-3 expression in 2-weeks-old Arabidopsis seedlings grown in 0.5x Hoagland optimal (ctrl), or sulfur-deficient (S-) or nitrogen-deficient (N-) medium assayed with semiquantitative RT-PCR. Tubuline (tua3) was used as quantity control.

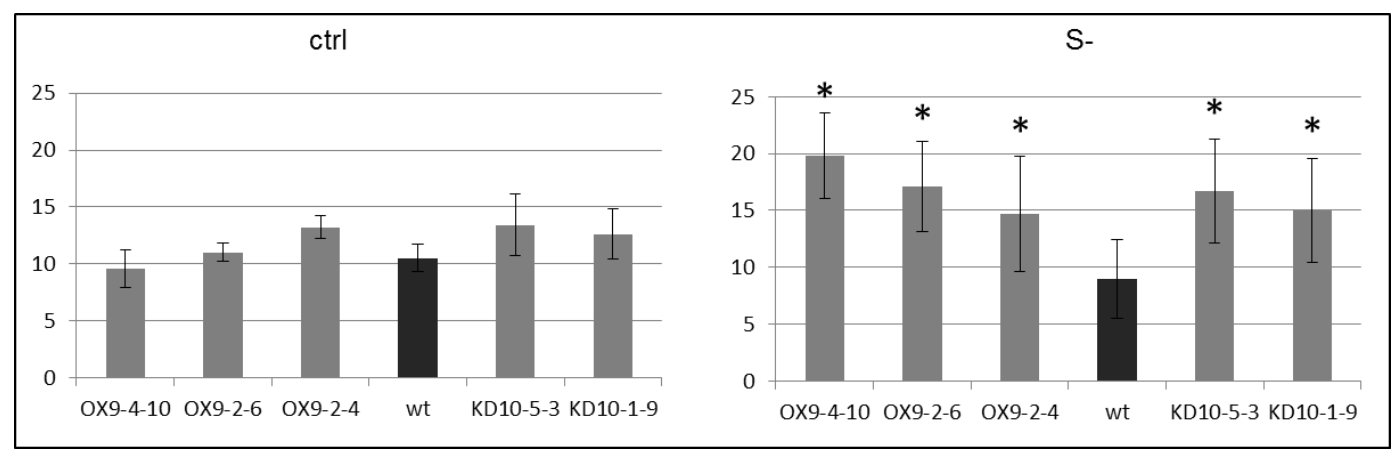

Fig. 4 The length of the roots of the seedlings grown for two weeks in 0.5x Hoagland medium, either optimal (ctrl) or sulfur deficient (S-). 


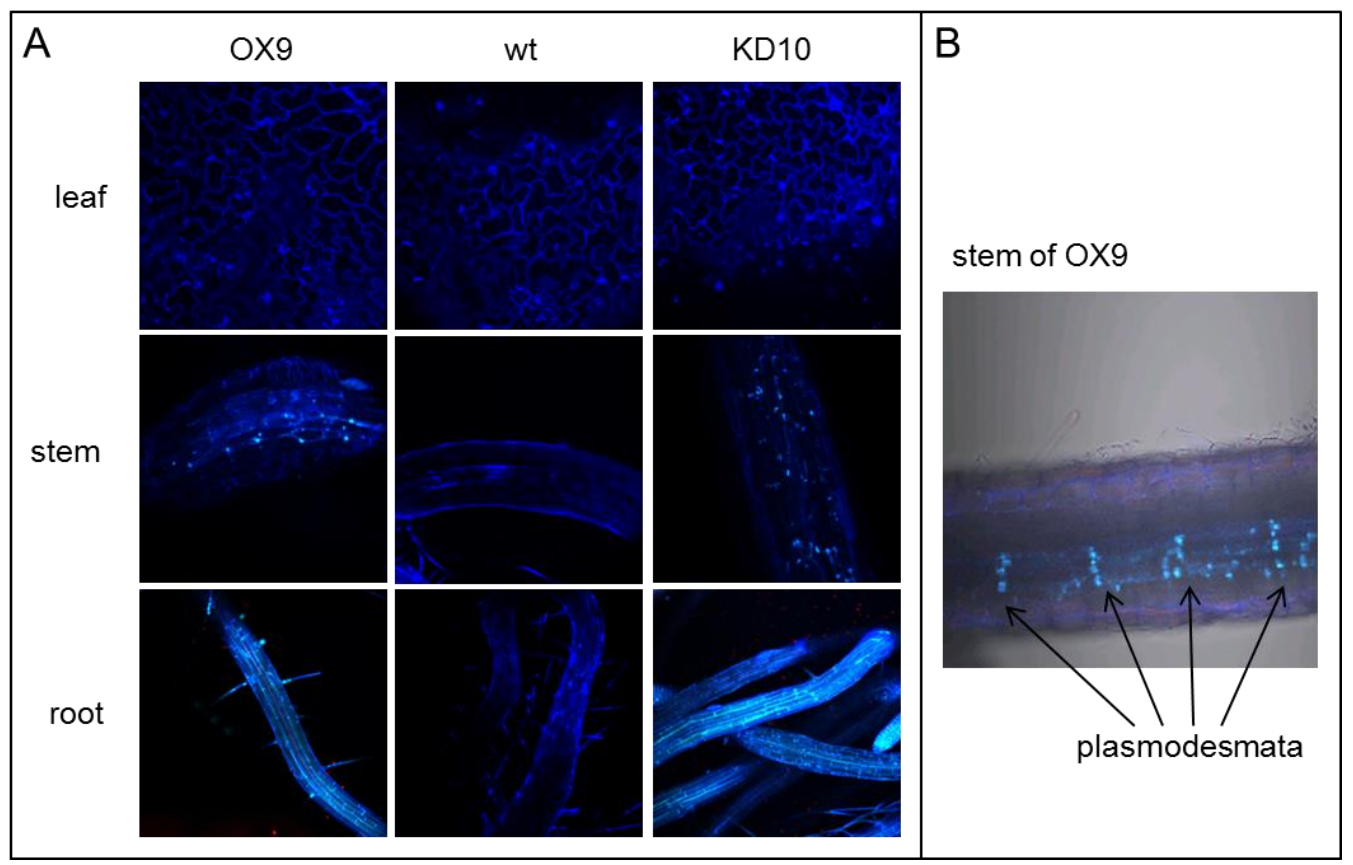

Fig. 5 Callose deposition in the 2-weeks-old Arabidopsis seedlings grown in 0.5x Hoagland optimal medium (wt, OX9-2-4 and KD10-1-9 line was used). The callose was stained with aniline blue for $1 \mathrm{~h}$ and next the tissues were observed under fluorescent confocal microscopy with $365 \mathrm{~nm}$ filter (1:40 magnification).

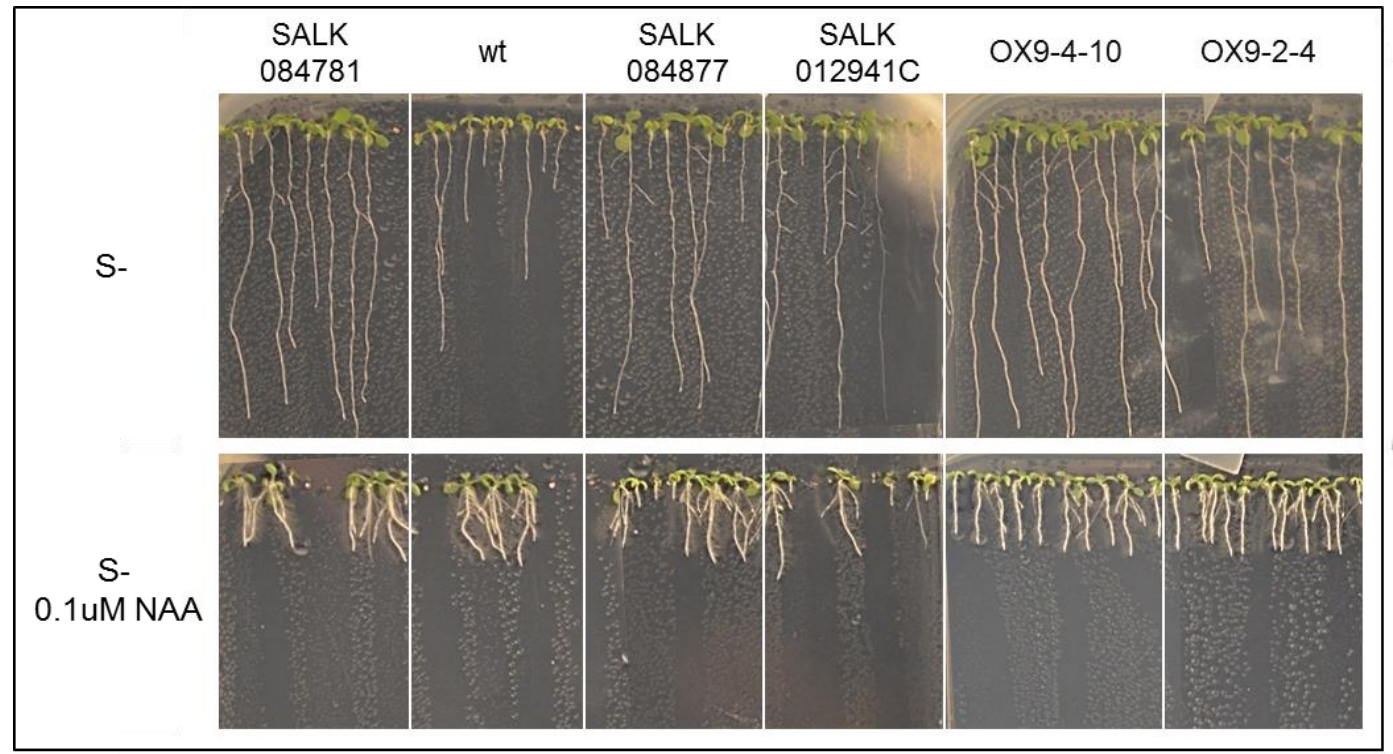

Fig. 6 Two-weeks-old Arabidopsis seedlings grown for two weeks in sulfur deficient 0.5x Hoagland medium (S-) with or without the addition of $0.1 \mu \mathrm{M}$ NAA. 